\title{
Search Engine Ad Auctions
}

\author{
Hal R. Varian \\ Google Inc. \\ University of California at Berkeley \\ hal@google.com
}

\begin{abstract}
Auctions for search engine advertising have been one of the most successful examples of economic mechanism design, at least in the private sector. This talk will review some of the history, theory, and practical issues surrounding these auctions.
\end{abstract}

